\title{
Revised FIGO staging system for cancer of the ovary, fallopian tube, and peritoneum: important implications for radiologists
}

\author{
Tsukasa Saida $^{1} \cdot$ Yumiko Oishi Tanaka $^{1} \cdot$ Koji Matsumoto $^{2} \cdot$ Toyomi Satoh $^{2}$ • \\ Hiroyuki Yoshikawa ${ }^{3} \cdot$ Manabu Minami $^{1}$
}

Received: 9 November 2015 / Accepted: 8 December 2015 / Published online: 22 December 2015

(C) Japan Radiological Society 2015

\begin{abstract}
Ovarian cancer is the seventh most common cancer diagnosis among women worldwide. The International Federation of Gynecology and Obstetrics recently significantly revised staging criteria for cancer of the ovary. The latest revision was based on the concept that high-grade serous tubal intraepithelial carcinoma (STIC) may be the origin of some high-grade serous carcinomas of the ovary and peritoneum. Therefore, staging criteria for the ovary, fallopian tube, and peritoneum have been unified. Understanding this background and other important revised points are essential for radiologists concerned with imaging diagnosis in gynecologic oncology. Through this review, we introduce the STIC theory and show examples of diseases in accordance with the new staging criteria based on magnetic resonance imaging (MRI) and computed tomography $(\mathrm{CT})$ results.
\end{abstract}

Keywords Staging - Ovarian cancer $\cdot$ Serous carcinoma . STIC

Tsukasa Saida

saida_sasaki_tsukasa@yahoo.co.jp

1 Department of Radiology, Faculty of Medicine, University of Tsukuba, 1-1-1 Tennodai, Tsukuba, Ibaraki 305-8575, Japan

2 Obstetrics and Gynecology, Faculty of Medicine, University of Tsukuba, 1-1-1 Tennodai, Tsukuba, Ibaraki 305-8575, Japan

3 Obstetrics and Gynecology, Ibaraki Prefectural Central Hospital, Koibuchi 6528, Kasama, Ibaraki 309-1793, Japan

\section{Introduction}

Ovarian cancer is the seventh most common cancer diagnosis among women worldwide and the fifth most common cancer diagnosis among women in higher-resource regions [1]. The International Federation of Gynecology and Obstetrics (FIGO) staging classification was revised in 1988 [2], and several subsequent scientific developments have challenged traditional concepts in ovarian cancer. The FIGO Committee on Gynecologic Oncology revised this classification in 2013 on the basis of these scientific developments in order to improve its utility and reproducibility [3]. Herein, we review the principal concepts of the revision and show some magnetic resonance imaging (MRI) and computed tomography (CT) examples for each disease stage.

\section{Tumor types of ovarian cancer}

Many histological types of malignant tumors arise from the ovaries, although approximately $90 \%$ of malignant ovarian tumors are of epithelial origin. These tumors are classified into five major subtypes: high-grade serous carcinoma (HGSC, $70 \%$ ), low-grade serous carcinoma (LGSC, $5 \%$ ), clear-cell carcinoma (CCC, $10 \%$ ), endometrioid carcinoma (EC, $10 \%$ ), and mucinous carcinoma (MC, $3 \%$ ) [3, 4]. Although the FIGO committee chose for simplicity a flexible staging system shared by all tumor types, a description of each histological subtype is required in the revised version.

\section{LGSC and HGSC}

Serous carcinoma (SC) has been classified into low and high grades on the basis of recent progress in 


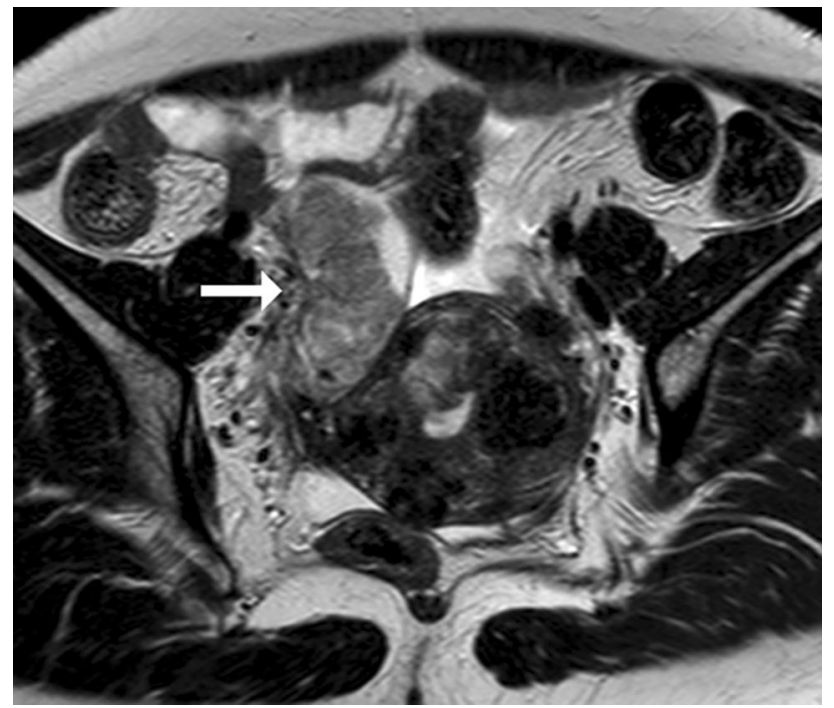

Fig. 1 A 48-year-old woman with low-grade serous carcinoma (LGSC). An axial T2-weighted magnetic resonance image (MRI) showing a solid mass composed of hyperintense papillary architecture with hypointense internal branching (arrow). Basic morphological characteristics of serous borderline tumors often remain in LGSC

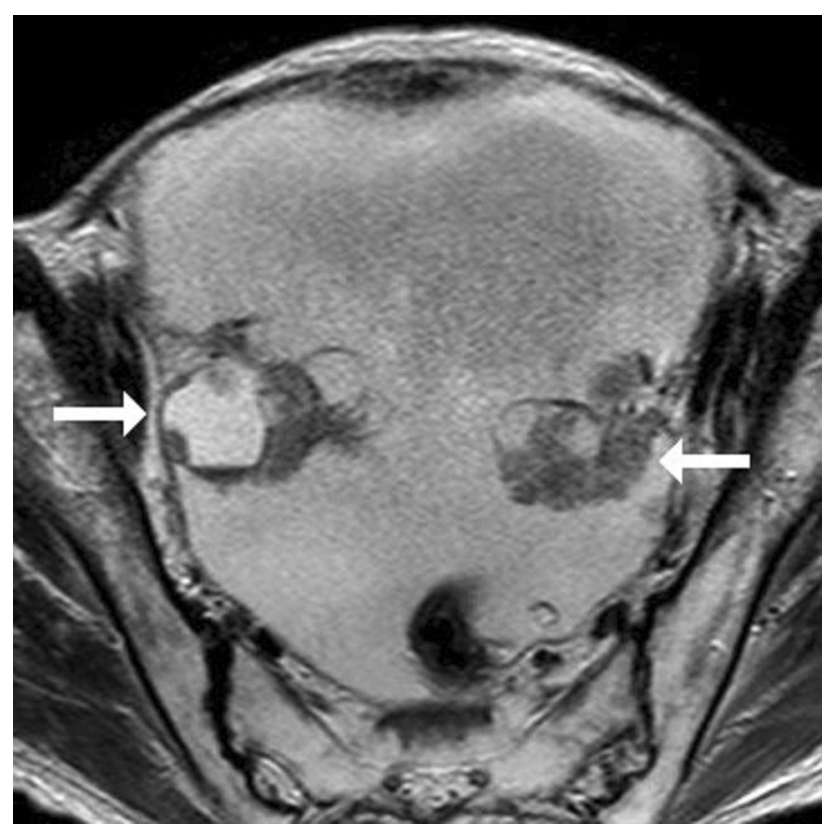

Fig. 2 A 64-year-old woman with high-grade serous carcinoma (HGSC). Bilateral ovarian masses appear as solid masses with cystic component on an axial T2-weighted magnetic resonance image (MRI) (arrows). Massive ascites caused by peritoneal metastases is also detected

immunohistochemical and molecular genetic analyses [59]. LSGC (Fig. 1) and HGSC (Fig. 2) are fundamentally different tumor types and even act as different diseases. LGSCs are thought to develop through a stepwise process

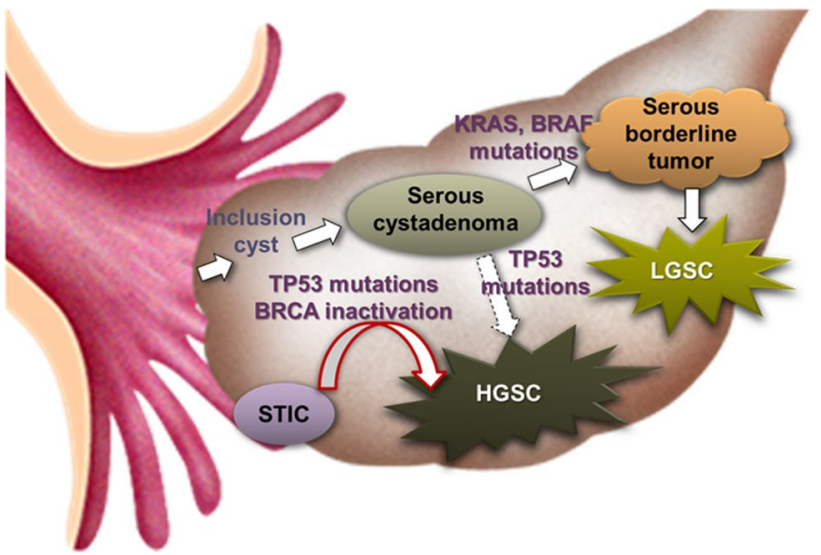

Fig. 3 A model of initiation and progression of low-grade (LGSC) and high-grade (HGSC) serous carcinomas. LGSC are considered to arise from serous borderline tumors in the stepwise process and are promoted by KRAS and BRAF mutations. HGSC are considered to be de novo cancers promoted by TP53 and BRCA abnormalities

from benign serous cystadenomas/adenofibromas to serous borderline tumors and ultimately to LGSCs, are promoted by $K R A S$ and $B R A F$ mutations and are unrelated to TP53 and $B R C A$ abnormalities [5-7]. It has been presumed that the precursors of serous cystadenomas/adenofibromas in this low-grade ovarian pathway are ovarian surface epithelial inclusion cysts $[5,6]$.

In contrast, HGSCs are considered to be de novo promoted by TP53 and BRCA abnormalities. In HGSCs, early loss of TP53 function followed by BRCA function loss leads to deficiency in homologous recombination repair of double-strand DNA (dsDNA) breaks. Subsequent events cause global changes in the gene that facilitates tumor evolution (Fig. 3) [5-9].

Definitiions of imaging findings of HGSC and LGSC have not yet been established. Serous borderline tumor, a precursor of LGSC, has been reported to appear as hyperintense papillary architecture with hypointense internal branching pattern on T2-weighted MRI [10, 11], and LGSC sometimes retains these characteristics. In contrast, HGSC is often seen proliferating in a monotonous manner forming a homogeneous solid adnexal masses [12] with carcinomatous peritonitis.

\section{An interpretation of the STIC theory}

Recently, the serous tubal intraepithelial carcinoma (STIC) theory has become widely accepted, and it may be an origin of HGSCs of the ovary and peritoneum [5-9]. Most ovarian cancer, especially HGSC, appear with extensive peritoneal dissemination and is often difficult to identify its origin. STIC develops into an invasive HGSC with rapid 


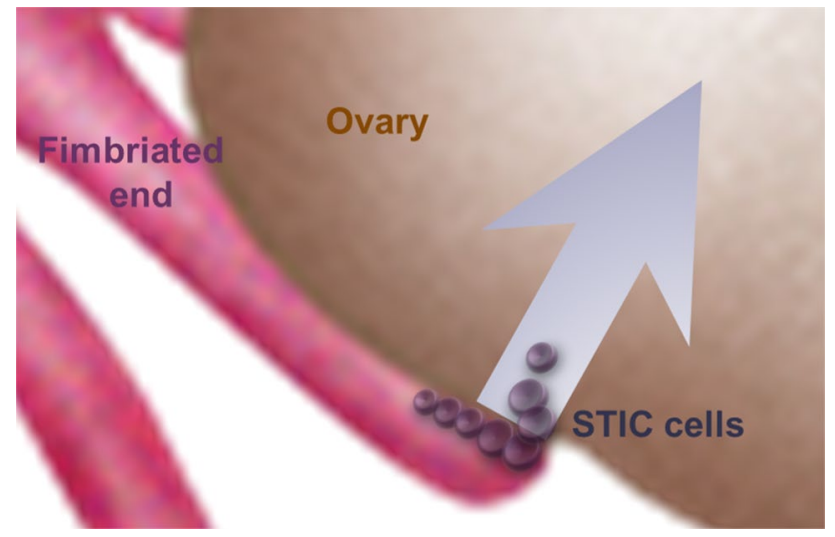

Fig. 4 Direct implantation of high-grade serous tubal intraepithelial carcinoma (STIC) cells onto the ovarian surface. Exfoliation of malignant cells from a high-grade STIC may implant on the ovarian surface and cause development of a high-grade serous carcinoma (HGSC) of the ovary

tumor growth and implants early onto the ovary or peritoneum. In the STIC theory, most HGSCs are believed to be of STIC origin.

The fimbria of the fallopian tube is normally in intimate contact with the ovarian surface at the time of ovulation. It is conceivable that when the ovarian surface epithelium is disrupted, normal tubal epithelial cells from the fimbria may be dislodged and implanted on the ovarian surface to form an inclusion cyst. Exfoliation of malignant cells from an STIC implant on the ovarian surface results in the development of an HGSC of the ovary (Fig. 4) [6].

According to this theory, there is no longer a reason for classifying HGSCs into ovarian, tubal, or peritoneal origin. Therefore, staging criteria of cancers of the ovary, fallopian tube, and peritoneum have been unified. STICs are often found in risk-reducing salpingo-oophorectomy specimens in BRCA-positive patients. In addition, TP53 mutations in both HGSC and STIC indicate a clonal relationship; HGSC shows similar immunoreactivity and secretory differentiation to that of STIC. These factors suggest that STIC is a precursor lesion of HGSC, and a distal fallopian tube is an important site for initiation of HGSC [5-9].

HGSC often appears as massive ascites, omental caking, peritoneal nodules, or enhancement, and these findings are dominant imaging features of carcinomatous peritonitis [13]. Before STIC theory was widely recognized, these diseases were known as Müllerian carcinomas. Müllerian carcinoma is defined as a malignant epithelial tumor of Müllerian duct origin, including ovaries, fallopian tubes, and peritoneum. Extensive peritoneal carcinomatosis may also originate from extragenital organs, such as stomach, colon, or breast. Therefore, radiologists must correctly differentiate metastatic ovarian tumors from HGSC.

\section{Revised FIGO staging criteria and key changes}

We compared the revised FIGO staging classification for cancer of the ovary, fallopian tube, and peritoneum (2013) and FIGO staging classification of the ovary (1988) [2] (Table 1).

\section{Stage I}

Stage I disease is confined to ovaries or fallopian tubes and peritoneal fluid/washings. There is no longer a need to distinguish cancers of ovarian origin from those of fallopian tube origin in stage I disease. However, the staging classification is almost the same as the former version of each ovarian and fallopian tube cancer. Tumors limited to one ovary or one fallopian tube are classified as stage IA (Fig. 5). Tumors limited to both ovaries or fallopian tubes are classified as stage IB (Fig. 6), which is relatively uncommon, accounting for only $1-5 \%$ of stage I cases [14, 15]. Tumor ruptures or those with surface involvement by tumor cells are classified as stage IC (Fig. 7). Microscopic spread of the tumor beyond adnexa is subdivided into stage IC1, IC2, or IC 3 by its cause (IC1, surgical spill; IC2, capsule ruptured before surgery or tumor on ovarian or fallopian tube surface; IC3, malignant cells in ascites or peritoneal washings). Therefore, the disease is diagnosed as stage IC only when apparent rupture of the tumor capsule is seen without nodular implants on the surface of the peritoneum or pelvic organs.

\section{Stage II}

Diseases limited to the pelvis are classified as stage II, regardless of whether the mass originates from ovaries or fallopian tubes. Primary peritoneal cancer is classified as stage II or greater. The primary site (i.e., ovary, fallopian tube, peritoneum) should be designated where possible. In some cases, it may not be possible to delineate the primary site clearly; such cases should be listed as undesignated. Extension and/or implants to the uterus and/or fallopian tubes and/or ovaries are subclassified as stage IIA (Fig. 8). Extension to other pelvic organs, such as colon or bladder, is subclassified as IIB (Fig. 9). Stage II disease only involves the peritoneum within the pelvic brim, whereas stage III disease can involve anywhere beyond the pelvic brim (Figs. 10, 11); there is a clear difference in terms of survival between these two diseases [3].

\section{Stage III}

Tumor spread to the peritoneum outside the pelvis and/or metastasis to the retroperitoneal lymph nodes is classified 
Table 1 Comparison of the revised International Federation of Gynecology and Obstetrics (FIGO) staging classification for cancer of the ovary, fallopian tube, and peritoneum (2013) and FIGO staging classification of the ovary (1988)

\begin{tabular}{|c|c|c|c|}
\hline \multicolumn{2}{|l|}{ FIGO (2013) } & \multicolumn{2}{|c|}{ FIGO (ovary, 1988) } \\
\hline I & Tumor confined to ovaries or fallopian tube(s) & I & Tumor limited to ovaries \\
\hline IA & $\begin{array}{l}\text { Tumor limited to } 1 \text { ovary (capsule intact) or fallopian } \\
\text { tube }\end{array}$ & IA & Tumor limited to 1 ovary \\
\hline IB & Tumor limited to both ovaries or fallopian tubes & IB & Tumor limited to both ovaries \\
\hline IC & $\begin{array}{l}\text { Tumor limited to } 1 \text { or both ovaries or fallopian tubes, } \\
\text { with any of the following }\end{array}$ & IC & $\begin{array}{l}\text { Tumor limited to } 1 \text { or both ovaries with any of the fol- } \\
\text { lowing: capsule ruptured, tumor on ovarian surface; } \\
\text { malignant cells in ascites }\end{array}$ \\
\hline IC1 & Surgical spill & $\mathrm{IC}(1 / 2)$ & Malignant cells in peritoneal washings/ascites \\
\hline IC2 & $\begin{array}{l}\text { Capsule ruptured before surgery or tumor on ovar- } \\
\text { ian or fallopian tube surface }\end{array}$ & $\mathrm{IC}(\mathrm{a} / \mathrm{b})$ & Capsule ruptured before surgery/surgical spill \\
\hline IC3 & Malignant cells in the ascites & & \\
\hline II & $\begin{array}{l}\text { Tumor involves } 1 \text { or both ovaries or fallopian tubes } \\
\text { with pelvic extension or primary peritoneal cancer }\end{array}$ & II & Tumor involves 1 or both ovaries with pelvic extension \\
\hline IIA & $\begin{array}{l}\text { Extension and/or implants on uterus and/or fallopian } \\
\text { tubes and/or ovaries }\end{array}$ & IIA & Extension and/or implants on uterus and/or tube(s) \\
\hline IIB & Extension to other pelvic intraperitoneal tissues & IIB & Extension to other pelvic tissues \\
\hline & & IIC & Pelvic extension with malignant cells in ascites \\
\hline III & $\begin{array}{l}\text { Tumor with spread to peritoneum outside the pelvis and/ } \\
\text { or metastasis to retroperitoneal lymph nodes }\end{array}$ & III & $\begin{array}{l}\text { Tumor with peritoneal metastases outside pelvis and/or } \\
\text { regional lymph node metastasis }\end{array}$ \\
\hline IIIA1 & Positive retroperitoneal lymph nodes only & IIIA & Microscopic peritoneal metastasis beyond pelvis \\
\hline IIIA1(i) & Metastasis $\leq 10 \mathrm{~mm}$ & & \\
\hline IIIA1(ii) & Metastasis $>10 \mathrm{~mm}$ & & \\
\hline IIIA2 & Microscopic extrapelvic peritoneal involvement & & \\
\hline IIIB & Macroscopic peritoneal metastasis beyond pelvis $\leq 2 \mathrm{~cm}$ & IIIB & Macroscopic peritoneal metastasis beyond pelvis $\leq 2 \mathrm{~cm}$ \\
\hline IIIC & Macroscopic peritoneal metastasis beyond pelvis $>2 \mathrm{~cm}$ & IIIC & $\begin{array}{l}\text { Peritoneal metastasis beyond pelvis }>2 \mathrm{~cm} \text { and/or } \\
\text { regional lymph node metastasis }\end{array}$ \\
\hline IV & Distant metastasis excluding peritoneal metastases & IV & Distant metastasis (excludes peritoneal metastasis) \\
\hline IVA & Pleural effusion with positive cytology & & \\
\hline IVB & $\begin{array}{l}\text { Parenchymal metastases and metastases to extraabdomi } \\
\text { nal organs }\end{array}$ & & \\
\hline
\end{tabular}

Key changes are indicated in boldface

as stage III. Exclusive retroperitoneal lymph node metastasis is classified as stage IIIA1 (Fig. 12) and no longer as stage IIIC because patients with lymph node involvement without peritoneal involvement have better prognosis [1621]. Less than $10 \%$ of ovarian carcinomas extend beyond the pelvis with exclusively retroperitoneal lymph node involvement [3]. Because lymphatic drainage of the ovary follows its venous supply by draining through the suspensory ligament to para-aortic lymph nodes and through the broad ligament to iliac chains, regional lymph nodes of ovarian/tubal/peritoneal carcinomas include obturator, internal, external, and common iliac nodes and para-aortic nodes below the renal hilum. On CT or MRI, lymph nodes with a short-axis diameter $>1 \mathrm{~cm}$ or clusters of smaller lymph nodes are considered to be metastases [22, 23]. On $\left[{ }^{18} \mathrm{~F}\right]$-fluorodeoxyglucose positron emission tomography (FDG-PET), diagnosis of metastatic lymph nodes is based on the presence of focal increased uptake of the tracer regardless of node size. The accuracy of detecting lymph node metastasis is reported to be higher in FDG-PET and FDG-PET/CT than in CT or MRI [15, 24, 25]. Sensitivity, specificity, and overall accuracy of PET or PET/CT were reported to be $73.2,96.7$, and $90.32 \%$ and of CT were 42.6, 95.0, and $19.87 \%$; those of MRI were 54.7, 88.3, and $12.38 \%$, respectively [24].

Microscopic extrapelvic peritoneal involvement is classified as stage IIIA2. It may appear on CT or MRI as massive ascites without implants. Macroscopic peritoneal metastasis beyond the pelvis of $\leq 2 \mathrm{~cm}$ in greatest dimension is classified as stage IIIB (Fig. 10); those $>2 \mathrm{~cm}$ in greatest dimension are classified as stage IIIC (Fig. 11). This is the same as the former staging classification. Tumor extension to capsules of the liver and spleen without parenchymal involvement of either organ is classified as stage IIIC and not IVB. HGSCs are often present in stage III, especially in stage IIIC (84\%) [5], and these tumors typically spread 


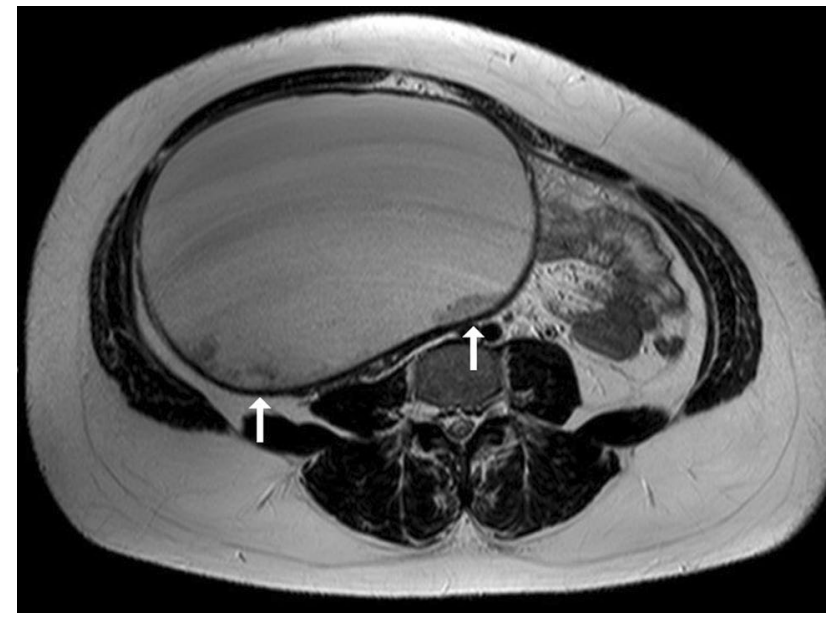

Fig. 5 A 28-year-old woman with serous borderline tumor stage IA. Large cystic tumor with multiple mural nodules replacing the right ovary is observed (arrow) on an axial T2-weighted magnetic resonance image (MRI). The tumor is limited to the unilateral ovary and is classified as stage IA

along peritoneal surfaces: omentum, bowel, mesentery, paracolic gutters, diaphragm, and serosal surfaces of liver and spleen. Among patients with stage IIIC or IV, survival after neoadjuvant chemotherapy followed by debulking surgery has been reported to have a similar survival rate as that after primary debulking surgery followed by chemotherapy [26]. Complete resection of all macroscopic disease at primary debulking surgery is the single most important independent prognostic factor, so radiologists should report details of all macroscopic peritoneal metastasis. Guidelines established by the European Society of Urogenital Radiology classify disease involving the following regions as being unresectable: retroperitoneal presacral disease, lymph node enlargement above the renal hilum, abdominal wall invasion, subcapsular liver metastases, and implants $>2 \mathrm{~cm}$ in diameter at diaphragm, lesser sac, porta hepatis, intersegmental fissure, gall bladder fossa, gastrosplenic, gastrohepatic ligament, and small bowel mesentery [22].

\section{Stage IV}

Generally, unresectable diseases-other than peritoneal metastasis and retroperitoneal lymph node metastasis-are designated as being stage IV. Stage IV disease, with malignant pleural effusion of positive cytology (Fig. 13), is subdivided into stage IVA and, with distant metastasis, into stage IVB (Fig. 14), which includes lymph node metastasis outside abdominal cavity: specifically, inguinal, supraclavicular, and cardiophrenic-angle lymph node metastasis. According to previous reports, cytologically proven
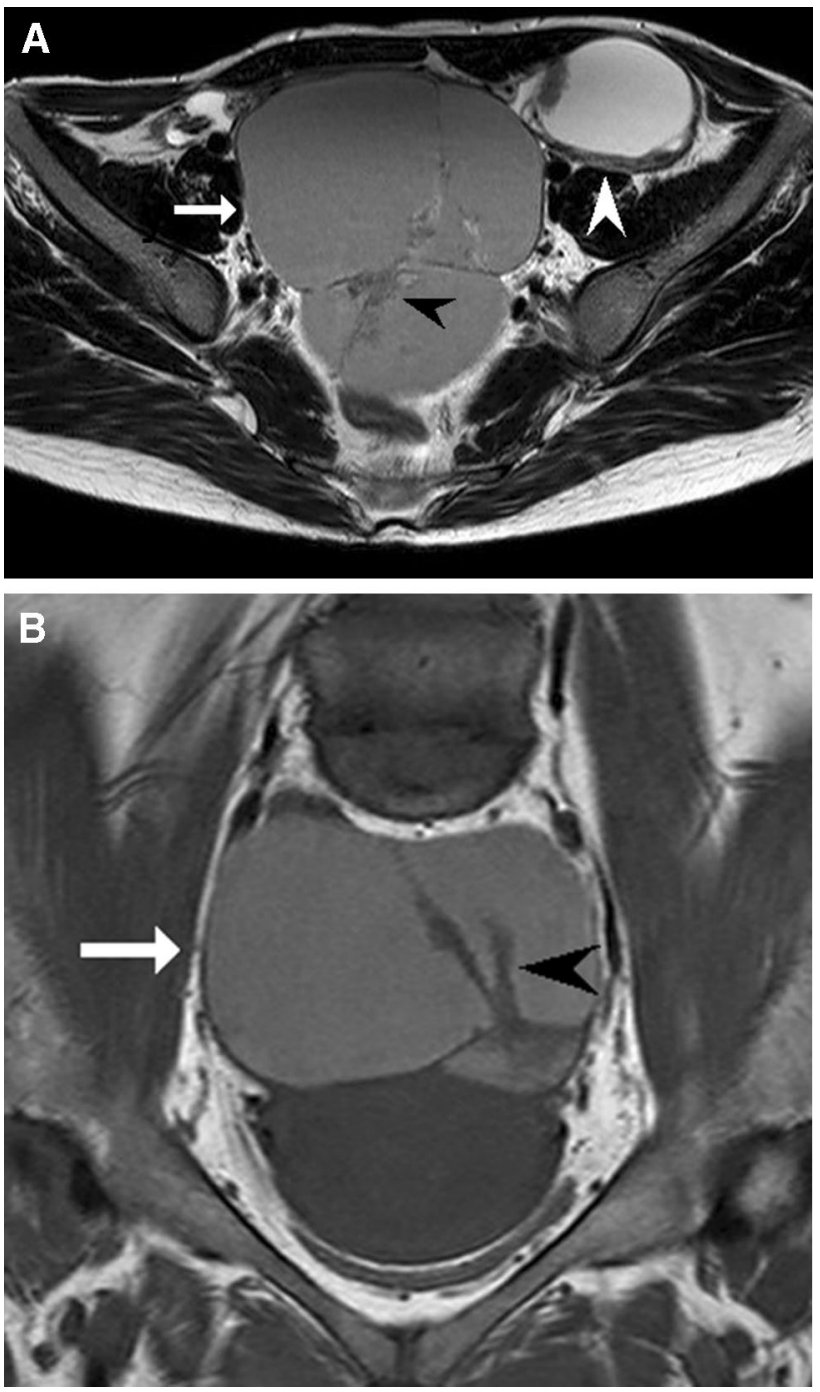

Fig. 6 A 41-year-old woman with endometrioid carcinoma, stage IB. a Cystic tumors (arrow and white arrowhead) with multiple mural nodules (black arrowhead) of both adnexa are observed on an axial T2-weighted magnetic resonance image (MRI). b Tumor on the right side shows slightly high signal intensity on a coronal T1-weighted MRI (arrow). This finding indicates that the tumor is associated with an endometriotic cyst. The tumors are limited to both ovaries and classified as stage IB

malignant pleural effusion has worse prognosis than that in stage IIIC [27] but better than that in stage IV disease [28]; therefore, this subdivision was added. Malignant pleural effusion may appear as massive pleural fluid and/or thickening of parietal pleura on CT or MRI. Transmural bowel infiltration, umbilical deposit, and parenchymal metastases in the liver and spleen or elsewhere are classified as stage IVB. Nevertheless, tumor extension from the peritoneum to the spleen or liver (stages IIIB or IIIC) should be differentiated from parenchymal metastases (stage IVB). 


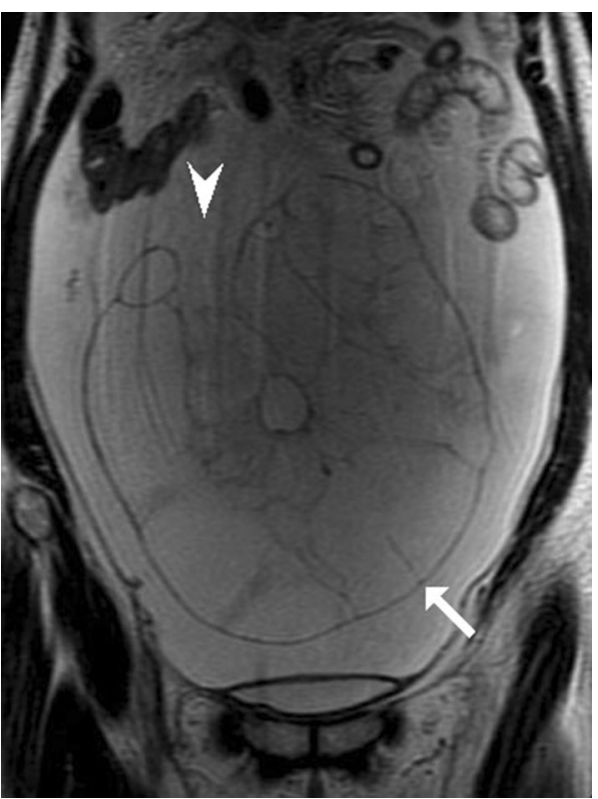

Fig. 7 A 66-year-old woman with mucinous borderline tumor with capsule rupture, stage IC. A large cystic tumor with multiple septa is observed (white arrow) on a coronal T2-weighted magnetic resonance image (MRI). A capsule defect (arrowhead) indicates rupture. Massive ascites are also observed. A tumor with ruptured capsule is classified as stage IC

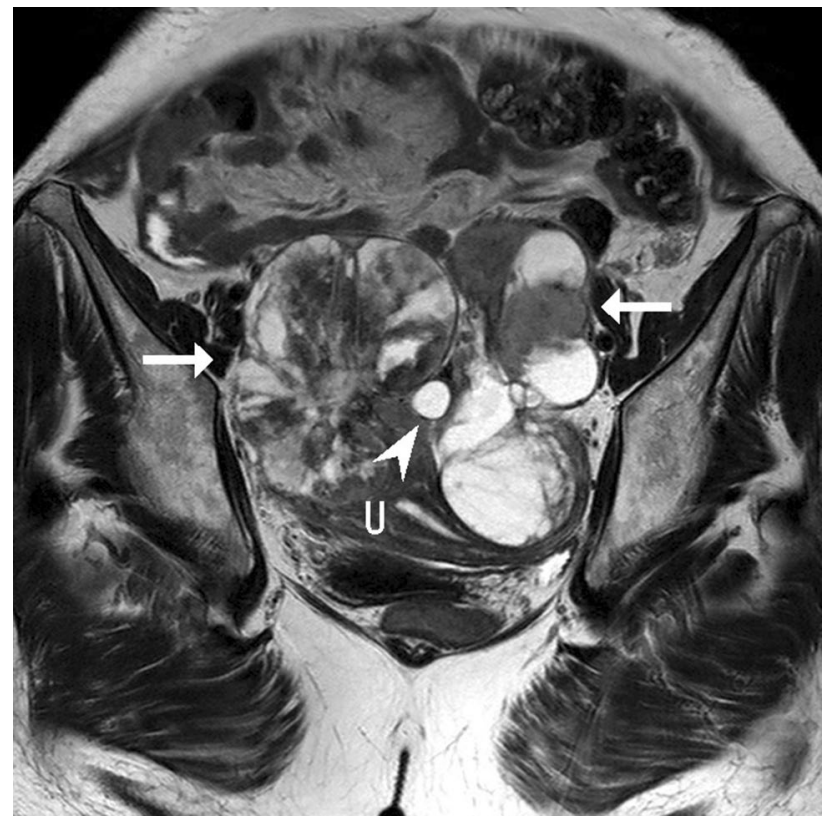

Fig. 8 A 74-year-old woman with mixed carcinoma of high-grade serous (HGSC) and clear-cell carcinoma, stage IIA. Solid and cystic tumors of both adnexa (arrows) invading the uterus $(U)$ and right fallopian tube (arrowhead) are clearly demonstrated on a coronal T2-weighted magnetic resonance image (MRI). Tumors with extension to the uterus and fallopian tubes are classified as stage IIA

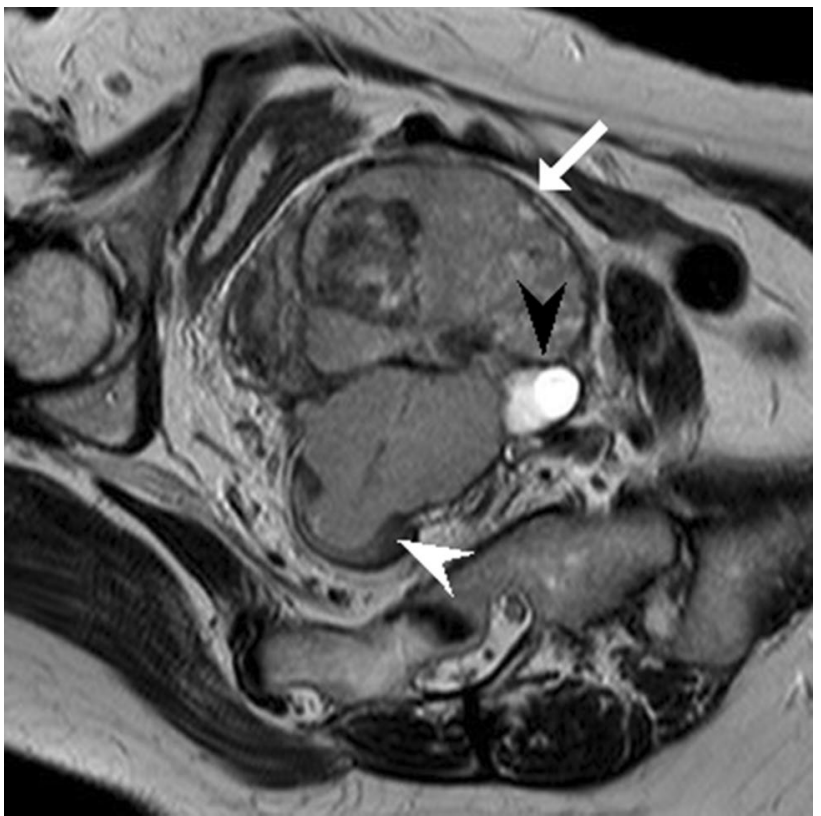

Fig. 9 A 63-year-old woman with high-grade serous carcinoma (HGSC), stage IIB. The rectum (white arrowhead) and the left fallopian tube (black arrowhead) show involvement with a solid tumor of the left adnexa (arrow) on an axial T2-weighted magnetic resonance image (MRI). Tumors with extension to the rectum are classified as IIB

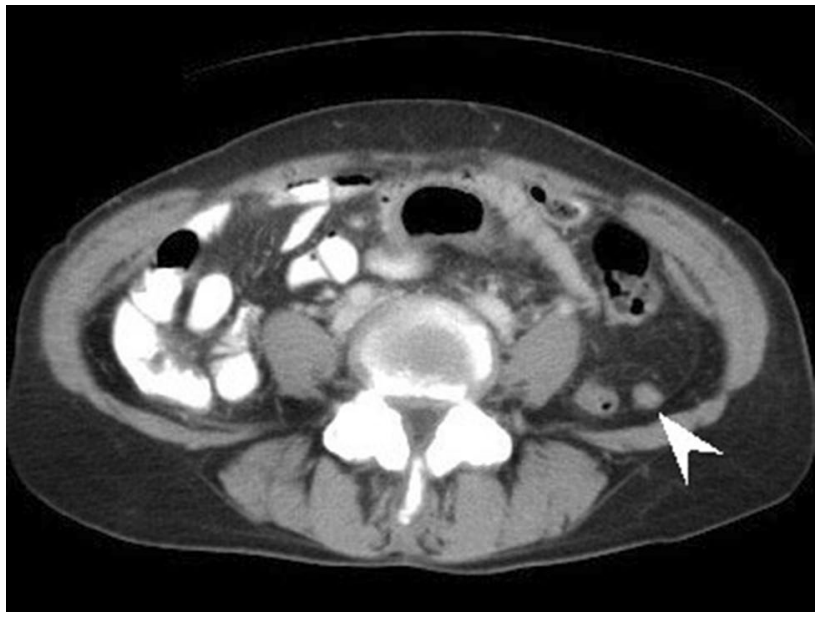

Fig. 10 A 57-year-old woman with high-grade serous carcinoma (HGSC), stage IIIB. Peritoneal metastases $\leq 2 \mathrm{~cm}$ (arrowhead) are observed in the left paracolic gutter beyond the pelvis on contrastenhanced computed tomography (CT) and are classified as stage IIIB 


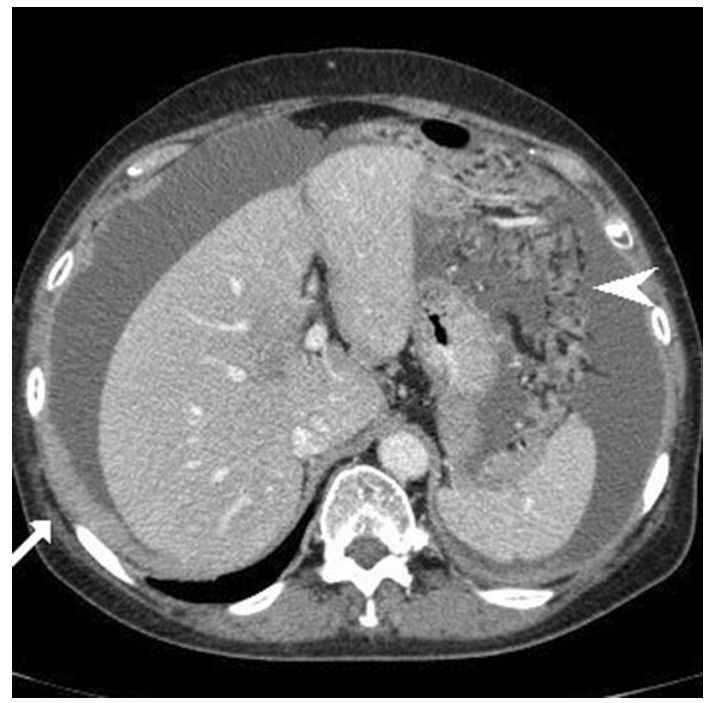

Fig. 11 A 66-year-old woman with high-grade serous carcinoma (HGSC), stage IIIC. HGSC causes sheet-like peritoneal implants in the right subphrenic space (arrow) and omental cake (arrowhead) $>2 \mathrm{~cm}$ in diameter on contrast-enhanced computed tomography (CT) and are classified as stage IIIC

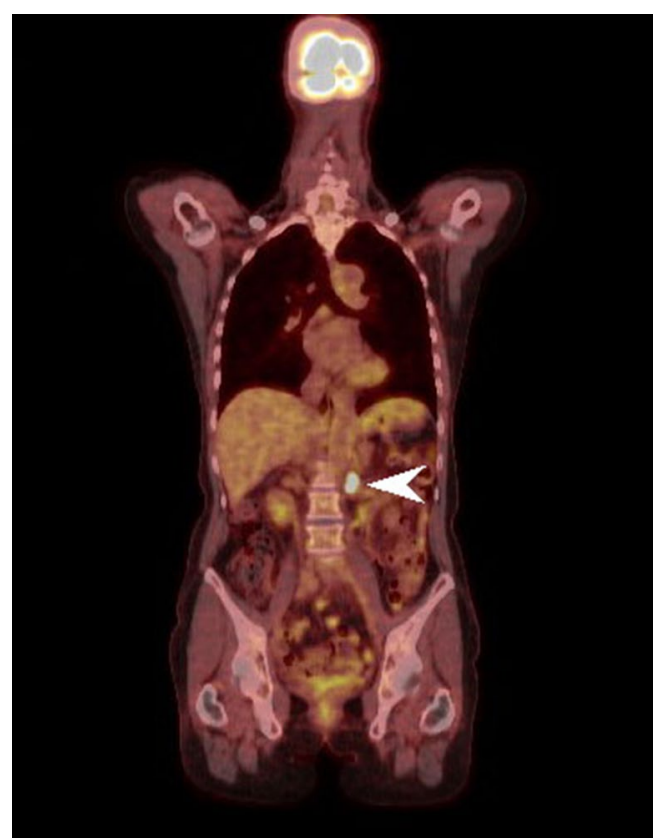

Fig. 12 A 55-year-old woman with mixed epithelial carcinomas, stage IIIA1. Para-aortic lymph node is revealed as a hot spot (arrowhead) with enlargement on a coronal $\left[{ }^{18} \mathrm{~F}\right]$-fluorodeoxy glucose positron emission tomography computed tomography (FDG-PET/CT), and peritoneal dissemination is not observed. Retroperitoneal lymph node metastasis alone is classified as stage IIIA1

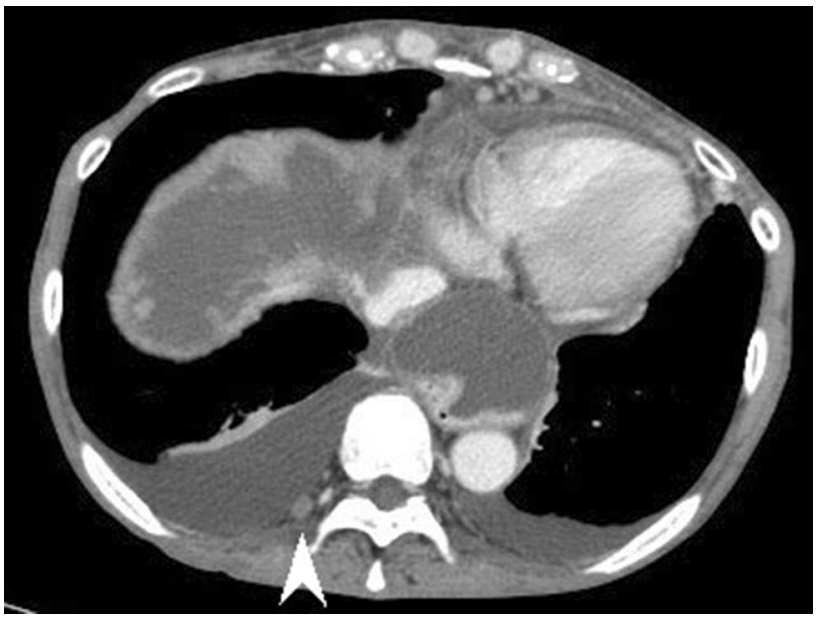

Fig. 13 A 64-year-old woman with high-grade serous carcinoma (HGSC), stage IVA. Contrast-enhanced computed tomography (CT) reveals bilateral pleural effusion with a tiny implant (arrowhead) on the surface of the parietal pleura. Malignant pleural effusion with positive cytology is classified as stage IVA

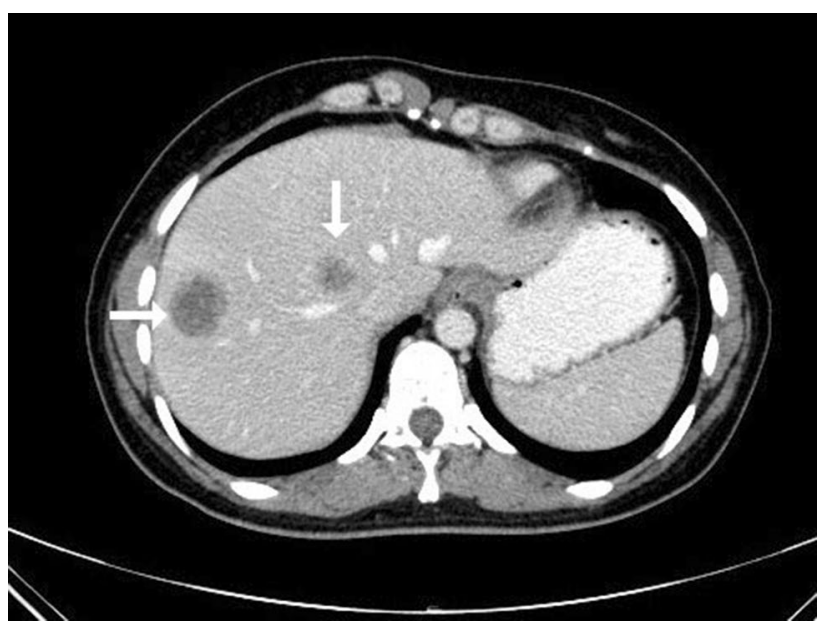

Fig. 14 A 45-year-old woman with high-grade serous carcinoma (HGSC), stage IVB. Liver metastases (arrows) are observed on contrast-enhanced computed tomography (CT) and are classified as stage IVB

\section{Conclusions}

Staging criteria of cancers of the ovary, fallopian tube, or peritoneum have been integrated according to the STIC concept. The following major changes from the previous criteria were made by the FIGO Committee on Gynecologic Oncology: Ovarian or tubal masses need no longer 
be distinguished from each other at stages I and II. Primary peritoneal cancer is defined as stage II. Exclusive lymph node metastasis is assigned to the new classification of stage IIIA1 and no longer to stage IIIC. Stage IV with malignant pleural effusion are subdivided into stage IVAand with distant metastases into stage IVB. Tumor extension from peritoneum to spleen or liver should be differentiated from parenchymal metastases (stage IVB).

\section{Compliance with ethical standards}

Conflict of interest The authors declare that they have no conflict of interest.

Ethical statement This article does not contain any studies with human participants or animals performed by any of the authors.

\section{References}

1. Ferlay J, Shin HR, Bray F, Forman D, Mathers C, Parkin DM. Estimates of worldwide burden of cancer in 2008: GLOBOCAN 2008. Int J Cancer. 2010;127:2893-917.

2. Heintz APM, Odicino F, Maisonneuve P, Quinn MA, Benedet JL, Creasman WT, et al. Carcinoma of the ovary. Int J Gynecol Obstet. 2006;95(Suppl 1):S163.

3. Prat J, FIGO Committee on Gynecologic Oncology. Staging classification for cancer of the ovary, fallopian tube, and peritoneum. Int J Gynaecol Obstet. 2014;124:1-5.

4. Kurman RJ, Carcangiu ML, Herrington S, Young RH. WHO classification of tumours of female reproductive organs. 4th ed. Lyon: IARC Press; 2014. p. 11-86.

5. Prat J. Ovarian carcinoma: five distinct disease with different origins, genetic alterations, and clinicopathological features. Virchows Arch. 2012;460:237-49.

6. Kurman RJ, Shih IeM. The origin and pathogenesis of epithelial ovarian cancer: a proposed unifying theory. Am J Surg Pathol. 2010;34:433-43.

7. Lalwani N, Prasad SR, Vikram R, Shanbhogue AK, Huettner PC, Fasih N. Histologic, molecular, and cytogenetic features of ovarian cancers: implications for diagnosis and treatment. Radiographics. 2011;31:625-46.

8. Gilks CB, Prat J. Ovarian carcinoma pathology and genetics: recent advances. Hum Pathol. 2009;40:1213-23.

9. Bowtell DD. The genesis and evolution of high-grade serous ovarian cancer. Nat Rev Cancer. 2010;10:803-8.

10. Tanaka YO, Okada S, Satoh T, Matsumoto K, Oki A, Nishida M, et al. Ovarian serous surface papillary borderline tumors form sea anemone-like masses. J Magn Reson Imaging. 2011;33:633-40.

11. Kim SH, Yang DM, Kim SH. Borderline serous surface papillary tumor of the ovary: MRI characteristics. AJR Am J Roentgenol. 2005;184:1898-900.

12. Bell DA, Scully RE. Early de novo ovarian carcinoma. A study of fourteen cases. Cancer. 1994;73:1859-64.

13. Morita H, Aoki J, Taketomi A, Sato N, Endo K. Serous surface papillary carcinoma of the peritoneum: clinical, radiologic, and pathologic findings in 11 patients. AJR Am J Roentgenol. 2004;183:923-8.
14. Heintz AP, Odicino F, Maisonneuve P, Quinn MA, Benedet JL, Creasman WT, et al. Carcinoma of the ovary: FIGO 26th annual report on the results of treatment in gynecological cancer. Int $\mathbf{J}$ Gynecol Obstet. 2006;95(Suppl 1):S161-92.

15. Yemelyanova AV, Cosin JA, Bidus MA, Boice CR, Seidman JD. Pathology of stage I versus stage III ovarian carcinoma with implications for pathogenesis and screening. Int J Gynecol Cancer. 2008;18:465-9.

16. Onda T, Yoshikawa H, Yasugi T, Mishima M, Nakagawa S, Yamada M, et al. Patients with ovarian carcinoma upstaged to stage III after systematic lymphadenctomy have similar survival to Stage I/II patients and superior survival to other Stage III patients. Cancer. 1998;83:1555-60.

17. Kanazawa K, Suzuki T, Tokashiki M. The validity and significance of substage IIIC by node involvement in epithelial ovarian cancer: impact of nodal metastasis on patient survival. Gynecol Oncol. 1999;73:237-41.

18. Panici PB, Maggioni A, Hacker N, Landoni F, Ackermann S, Campagnutta E, et al. Systematic aortic and pelvic lymphadenectomy versus resection of bulky nodes only in optimally debulked advanced ovarian cancer: a randomized clinical trial. J Natl Cancer Inst. 2005;97:560-6.

19. Cliby WA, Aletti GD, Wilson TO, Podratz KC. Is it justified to classify patients to Stage IIIC epithelial ovarian cancer based on nodal involvement only? Gynecol Oncol. 2006;103:797-801.

20. Ferrandina G, Scambia G, Legge F, Petrillo M, Salutari V. Ovarian cancer patients with "node-positive-only" Stage IIIC disease have a more favorable outcome than Stage IIIA/B. Gynecol Oncol. 2007;107:154-6.

21. Baek SJ, Park JY, Kim DY, Kim JH, Kim YM, Kim YT, et al. Stage IIIC epithelial ovarian cancer classified solely by lymph node metastasis has a more favorable prognosis than other types of stage IIIC epithelial ovarian cancer. J Gynecol Oncol. 2008;19:223-8.

22. Forstner R, Sala E, Kinkel K, Spencer JA. ESUR guidelines: ovarian cancer staging and follow-up. Eur Radiol. 2010;20:2773-80.

23. Mitchell DG, Javitt MC, Glanc P, Bennett GL, Brown DL, Dubinsky T, et al. American College of Radiology. ACR appropriateness criteria staging and follow-up of ovarian cancer. J Am Coll Radiol. 2013;10:822-7.

24. Yuan Y, Gu ZX, Tao XF, Liu SY. Computer tomography, magnetic resonance imaging, and positron emission tomography or positron emission tomography/computer tomography for detection of metastatic lymph nodes in patients with ovarian cancer: a meta-analysis. Eur J Radiol. 2012;81:1002-6.

25. Kitajima K, Murakami K, Yamasaki E, Kaji Y, Fukasawa I, Inaba N, et al. Diagnostic accuracy of integrated FDG-PET/contrast-enhanced CT in staging ovarian cancer: comparison with enhanced CT. Eur J Nucl Med Mol Imaging. 2008;35:1912-20.

26. Vergote I, Tropé CG, Amant F, Kristensen GB, Ehlen T, Johnson $\mathrm{N}$, et al. Neoadjuvant chemotherapy or primary surgery in stage IIIC or IV ovarian cancer. N Engl J Med. 2010;363:943-53.

27. Eitan R, Levine DA, Abu-Rustum N, Sonoda Y, Huh JN, Franklin CC, et al. The clinical significance of malignant pleural effusions in patients with optimally debulked ovarian carcinoma. Cancer. 2005; 103:1397-401.

28. Aletti GD, Podratz KC, Cliby WA, Gostout BS. Stage IV ovarian cancer: disease site-specific rationale for postoperative treatment. Gynecol Oncol. 2009;112:22-7. 